\title{
Eli Lederhendler, Who Owns Judaism? Public Religion and Private Faith in America and Israël
}

Studies in Contemporary Jewry, vol. XVII, Oxford, Oxford University Press, The Institute of Contemporary Jewry, The Hebrew University of Jerusalem, 2001, 299 p.

\section{Chantal Bordes-Benayoun}

\section{(2) OpenEdition}

\section{Édition électronique}

URL : http://journals.openedition.org/assr/1256

DOI : $10.4000 /$ assr. 1256

ISSN : $1777-5825$

Éditeur

Éditions de l'EHESS

\section{Édition imprimée}

Date de publication : 1 avril 2003

Pagination : 59-157

ISBN : 2-222-96732-5

ISSN : 0335-5985

Référence électronique

Chantal Bordes-Benayoun, «Eli Lederhendler, Who Owns Judaism? Public Religion and Private Faith in America and Israël », Archives de sciences sociales des religions [En ligne], 122 | avril - juin 2003,

document 122.31, mis en ligne le 10 novembre 2005, consulté le 21 septembre 2020. URL : http:// journals.openedition.org/assr/1256 ; DOI : https://doi.org/10.4000/assr.1256 
La pertinence novatrice de telles notations illustre le fait que le parti-pris d'objectivation à partir de la matérialité des données ne bride pas chez l'A. l'intuition des dimensions proprement psychiques. Il accepte fort bien que «l'attention flottante " propre à l'écoute analytique ait sa propre fécondité en explorant le champ de l'inconscient, par exemple dans l'interprétation des rêves et plus généralement des textes thérésiens narratifs; mais il choisit professionnellement la restriction ascétique au sens obvie de chaque phrase, démarche qui donne également à entendre des processus psychologiques restés inaperçus jusqu'ici.

Les études thérésiennes sont entrées dans une ère nouvelle. L'œuvre de C.L. est déjà assez avancée pour avoir changé notre horizon. Quelle que soit la discipline (scientifique ou théologique) qu'on mette en œuvre pour faire avancer les études thérésiennes, il ne sera plus possible d'aborder le dossier comme avant la publication des trois volumes déjà parus.

De surcroît, l'A. manifeste un tel respect pour la diversité des disciplines que le dialogue interdisciplinaire s'en trouve facilité. Par exemple, une démarche psychanalytique traiterait à sa manière chez Thérèse le désir d'accéder au sacerdoce. Celui-ci serait envisagé comme héritage du désir qui tenaillait sa mère : avoir un fils prêtre. Dans l'économie psychique propre à Thérèse, ce désir, centré sur le maternage de Jésus par Marie, pourrait suggérer deux interprétations. Pour la première période de sa vie monastique, on entendrait un fantasme de restauration du corps à corps mère-bébé. Puis l'apaisement dans la fraternisation avec un missionnaire prendrait éventuellement le sens d'une satisfaction enfin donnée au désir maternel, de sorte que Thérèse peut enfin assumer son propre désir en cessant de se vivre comme décevante parce que fille (donc écartée du sacerdoce). Nous côtoyons ici la conclusion suggérée par C.L. (p. 175).

Tout chercheur qui travaille sur le dossier de Thérèse, quelle que soit sa spécialité, devra rester attentivement réceptif à la suite des publications annoncées par l'A. ...

Jacques Maître.

LEDERHENDLER (Eli), ed.

Who Owns Judaism? Public Religion and Private Faith in America and Israël. Studies in Contemporary Jewry, vol. XVII, Oxford, Oxford University Press, The Institute of Con- temporary Jewry, The Hebrew University of Jerusalem, 2001, 299 p.

À qui appartient le judaïsme ? C'est à cette question qui semble indiquer d'emblée une controverse, et peut-être un conflit d'intérêts, que la revue Studies in Contemporary Jewry consacre son XVIIe numéro. Si la question de l'identité juive est aujourd'hui devenue banale, de même que la diversité des réponses qui lui sont généralement apportées, le propos de l'ouvrage est autre. Il ne se situe pas sur le terrain de l'identité culturelle, de la judéité, mais bien sur celui du judaïsme lui-même. Peut-on véritablement parler d'un renouveau? Quels rapports les judaïcités contemporaines ont-elles à leur religion aux États-Unis et en Israël ? Quel statut lui accordent-elles dans leurs pratiques quotidiennes, leurs revendications dans la cité et leurs visions du monde? Quelle est donc aujourd'hui la place du judaïsme, de ses textes, de sa tradition, et de sa théologie pour les juifs et à l'intérieur des sociétés concernées ?

Les diverses contributions réinterrogent une tradition de recherche qui avait jusque-là privilégié le rôle de l'affiliation ethnique et accordé une moindre importance aux croyances et pratiques religieuses. Dans son introduction générale, l'éditeur principal du volume, E.L. signale ces enjeux de connaissance en montrant le renversement de perspective qui s'est opéré à partir de la seconde moitié du $\mathrm{XX}^{\mathrm{e}}$ siècle. Alors que les décennies de la sécularisation ont accrédité l'idée qu'elle n'était pas la condition sine qua non de la perpétuation du peuple juif, la religion semble aujourd'hui «reprendre ses droits » et partant, ne pas être un simple attribut, parmi d'autres, de l'ethnicité. La référence religieuse, comme mythe collectif fondateur et mobilisateur, tend à recouvrer une place centrale, indispensable même, suggèrent certains des auteurs, au sein d'une culture conçue comme un tout. Les chemins de ce retour vers la sphère religieuse sont multiples et suscitent divers conflits d'interprétation. Sur le plan théorique, le volume verse donc d'appréciables éléments au débat sur les rapports entre religion et ethnicité.

Plusieurs sources d'information sont alors exploitées pour ce dossier, concernant l'évolution théologique et rituelle, l'organisation communautaire, les pratiques et représentations quotidiennes. La lutte pour la modernisation religieuse semble s'être intensifiée au cours des dernières décennies qui ont été marquées aux États-Unis par une avancée significative en matière de conversion, de droits des femmes avec l'appropriation progressive par celles-ci d'une partie des domaines rituels habituellement dévolus aux hommes, et ce malgré le 
maintien de certaines discriminations, en matière de recrutement clérical par exemple (cf. les articles de Sylvia Barack Fishman, et de Allan Silverstein). De même l'évolution concernant l'homosexualité a progressivement rejoint le mouvement de rénovation initié par les Églises protestantes dans les années quatrevingt. En revanche, des traditionalistes aux plus libéraux, tous les courants du judaïsme se montrent à des degrés divers très réticents à l'égard des mariages mixtes, pierre angulaire de l'identité et de la perpétuation. Le devoir d'endogamie est d'autant plus réaffirmé par les responsables religieux que la réalité sociologique de l'exogamie touche plus de quarante pour cent de la population. Contre toute attente, les plus farouches opposants au mariage mixte ne se trouvent pas nécessairement dans les rangs des plus conservateurs mais chez certains « libéraux »- le mouvement dit conservative-, réputés ouverts mais inquiets de l'érosion de la fidélité juive qui en résulte. La redistribution des idées sur le terrain théologique, ainsi qu'un important renouvellement philosophique (par exemple la question de l'au-delà et de la résurrection analysée par Gillman) tendent à brouiller les anciens clivages pour redéfinir une norme collective et légitime, sous l'action croisée et contradictoire des divers acteurs institutionnels et individuels. Pour sa part, la société israélienne est également et d'autant plus travaillée par cette quête de redéfinition de l'espace du religieux, que les dimensions nationale et religieuse sont ici profondément intriquées (articles de Inbari et Ratzabi). Dans les deux aires étudiées -même si le petit nombre d'articles sur le cas israélien n'autorise pas toujours la comparaison -, une tension permanente entre la nécessité de l'action collective, la volonté et la liberté individuelles semble travailler à l'infini la production d'une référence commune définissant le judaïsme pour les juifs mais aussi pour les non juifs.

Quête improbable sinon impossible, cette recherche d'authenticité s'effectue à la fois dans l'intimité du foyer et de plus en plus dans l'espace public. Aussi les enquêtes de terrain et les faits sont-ils importants pour en apprécier l'occurrence. Ils témoignent en premier lieu de l'affirmation de valeurs individuelles qui, audelà des cas de figure présentés, appartiennent à un ensemble de valeurs et d'intérêts proclamés sur une plus large échelle, dans la société globale. Dans une enquête menée auprès d'un millier de personnes, Steven M. Cohen établit le poids effectif du fait religieux, conçu comme pratique, comme référent identitaire et comme base d'affiliation, chez les juifs américains dans leur ensemble et parmi les plus jeunes en particulier. Il note que cette appropriation religieuse s'effectue dans un contexte d'effondrement des fondements sociaux de l'ethnicité tels que la proximité spatiale des membres de la communauté, les réseaux de sociabilité et l'endogamie. Plusieurs contributions s'accordent à constater l'affaiblissement des cadres et références communautaires au profit d'une atomisation des formes de fidélité. Dans l'état d'esprit qualifié de " postmoderne» qui en résulte, semblent triompher des formes variées et souvent contradictoires sinon conflictuelles : le « romantisme religieux », le rejet de l'adaptation au rationalisme moderne qui avait prévalu dans le monde juif depuis l'ère de l'Émancipation, l'importance accordée à l'exil spirituel plutôt qu'à l'histoire diasporique (Ratzabi, Inbari), la recherche de spiritualité, le questionnement mystique, la protestation, la valorisation du recueillement plutôt que l'action, l'attrait pour d'autres formes de spiritualité, la liberté individuelle de choix et d'action dans tous ces domaines. Autant de déclinaisons individuelles qui redessinent les différentiations et conflits internes, comme le montre à l'extrême, l'exemple de mouvements apocalyptiques qui vont jusqu'à désigner un complot interne au peuple d'Israël (dans la secte Uzi Meshulam étudiée en Israël par Inbari, par exemple). Si une telle variété est possible, c'est que la liberté individuelle de choix et d'obédience existe dans les sociétés démocratiques. Dès lors les " groupes religieux sont en compétition avec d'autres sur un marché dans lequel les individus trouvent la forme de religion qui convient le mieux à leurs penchants » (Ezra Kopelowitz).

Paradoxe de ces valorisations individuelles et privées, elles se produisent dans, et peut-on ajouter sont encouragées par, l'espace public, où elles ont pris une importance considérable à la fin du $\mathrm{XX}^{\mathrm{e}}$ siècle. Et sans doute trouve-t-on ici une partie de la réponse que les auteurs apportent à la question de départ : le judaïsme appartient en quelque sorte au domaine public. Le symbole de cette présence publique du judaïsme religieux, souligne David Ellenson, est par exemple incarné par J. Lieberman, candidat à la prochaine élection à la Maison Blanche qui affiche fortement son identité de croyant-pratiquant. L'affirmation juive prend un tour différent de celles qui avaient précédemment prévalu en particulier dans la gauche américaine. On réservait à la judéité un sens plutôt ethnique que religieux et on en promouvait une image laïque. Le succès public de cette modalité religieuse d'expression de la judéité n'est compréhensible que parce qu'il répond à une demande de religiosité de la société tout 
entière. Si l'on manque d'éléments de comparaison avec d'autres courants religieux du paysage américain, les AA. ont évoqué à maintes reprises des faits symétriques dans le monde protestant. C'est une évolution globale de la légitimité de la foi et du culte qui semble ici à 1'œuvre dans des sociétés démocratiques où le religieux suscite sans doute d'analogues engouements et de comparables défiances.

Chantal Bordes-Benayoun.

\subsection{2}

LEE (Thomas H.C.).

Education in Traditional China. An History. Leyde (Pays-Bas), Brill, 2000, xiv + 762 p. (bibliogr., index, liste des caract. chinois) (coll. "HdO. Handbook of Oriental Studies/ Handbuch der Orientalistik ", section 4, China, 13).

Ayant, depuis vingt-cinq à trente ans, centré sa carrière orientaliste sur l'étude de l'éducation dans la Chine classique et sur le sentiment que, contrairement à l'idée longtemps admise, le confucianisme n'a pas été cause d'un retard sur le chemin de la modernisation, l'auteur (qui publie aussi en chinois sous le nom de $\mathrm{Li}$ Hongqi) se livre ici à l'exercice périlleux de rédiger seul un manuel de référence, là où on attendrait toute une équipe conviée autour d'un maître d'œuvre, et la somme qu'il réalise est impressionnante. Les théories sous-jacentes sont, d'une part, la complexité du système d'éducation, qu'il est impossible de réduire au seul confucianisme, un «caméléon » (p. 40), alors que s'entremêlent, entre autres, l'influence des pratiques religieuses populaires et des traditions locales, et, d'autre part, la valeur intrinsèque d'une telle éducation et le plaisir qu'elle procurait à ceux qui en bénéficiaient. Cinq chapitres thématiques déroulent l'un après l'autre leurs arguments selon un ordre chronologique qui, malheureusement s'arrête à la fin des Ming au milieu du $\mathrm{XVII}^{\mathrm{e}}$ siècle, sans qu'une telle limitation soit annoncée dans le titre de l'ouvrage.

L'introduction (chap. 1) survole les idéaux de l'éducation chinoise : le perfectionnement de soi-même en même temps qu'une implication sociale, le tout modelé par l'idéologie confucianiste dont le conservatisme a contribué à maintenir la stabilité du monde chinois. Au chapitre de l'histoire institutionnelle (chap. 2), sont, en première partie, traités les différents types d'écoles, dynastie après dynastie depuis l'Antiquité - institutions impériales, privées, locales, de niveau élémentaire, moyen, supérieur ou académique, avec leurs objectifs propres et les difficultés auxquelles elles se sont heurtées; sous les Sui et les Tang, à partir du $\mathrm{VII}^{\mathrm{e}}$ siècle, l'éducation passe à l'ombre du temple confucéen local et le système se répand en Corée, au Vietnam et au Japon ; à partir des Song au $\mathrm{X}^{\mathrm{e}}$ siècle, l'enseignement devient le marche-pied conduisant aux examens d'État; sous les dynasties de conquête des Jin (Jürchen) et des Yuan (Mongols) aux XII ${ }^{\mathrm{e}}$-XIV ${ }^{\mathrm{e}}$ siècles, le système éducatif doit prendre en charge aussi l'enseignement de la langue de la classe dirigeante non-chinoise, tandis que chez les autochtones se développe un sentiment national chinois ; à la dynastie mongole, revient l'introduction d'écoles de communautés (shexue) destinées à atteindre l'ensemble de la jeunesse chinoise en âge d'être scolarisée et qui, sous les Ming à partir de la fin du XIV e siècle, deviennent universelles; les sociétés intellectuelles locales et les académies sont d'ailleurs, à cette époque, fortement impliquées dans l'éducation populaire, mais l'autoritarisme gouvernemental, de plus en plus marqué, interdit tout activisme un tant soit peu politique. La seconde partie de ce même chapitre 2 analyse la genèse du système des examens introduisant aux services civils qui ont fait la gloire de la Chine impériale, et les programmes en sont donnés pour les Han et les Tang; même les dynasties de conquête jürchen et mongole ont appuyé le système (les Mongols à partir de 1314), avec un cursus et des quotas pour les étrangers distincts de ceux des Chinois ; l'on doit aux Yuan l'affirmation définitive de l'orthodoxie néo-confucéenne défendue dans les examens sur la base des commentaires que Zhu Xi (1130-1200) a apportés aux vénérables classiques de l'Antiquité; et aux Ming la pratique des essais formels dits « à huit membres », l'absolutisme des Ming contribuant, en outre, à renforcer le rôle des examens dans la formation d'une méritocratie orthodoxe.

Le chapitre 3 brosse l'ensemble du développement de la pensée chinoise dans tous ses aspects, depuis Confucius et les sages de l'Antiquité, la transmission des textes classiques, les interférences bouddhistes et taoïstes, l'idéal aristocratique de l'éducation familiale, les programmes universitaires aux $\mathrm{IV}^{\mathrm{e}}-\mathrm{VI}^{\mathrm{e}}$ siècles, l'apparition de nouvelles disciplines comme l'histoire et la littérature, jusqu'à la standardisation et la codification des cinq Classiques et des rites gérant la construction de l'orthodoxie sous les Song, jusqu'au prosélytisme populaire de haut niveau ou à l'antinomie entre une éducation néo-confucéenne utilitariste et l'érudition pure, ou encore à la constitution de la théorie des "Trois Enseignements" unifiés - confucianisme, bouddhisme, taoïsme. L'éducation néo-confucéenne est détaillée depuis la découverte de l'enfance, une caractéristique des Song, jusqu'à la formation des maî- 